\title{
Avaliação da interferência do ácido ascórbico na detecção da glicosúria
}

\author{
Evaluation of ascorbic acid interference in the detection of glycosuria
}

Jaime Murilo Fernandes Costa'; Maria Elizabete Mendes²; Nairo Massakazu Sumita ${ }^{3}$

unitermos
Ácido ascórbico
Glicosúria
Química seca
Interferência

\section{resumo}

Introdução: O ácido ascórbico (vitamina C) é comumente ingerido como suplemento vitamínico. É uma vitamina hidrossolúvel, excretada pela urina e pode interferir nos ensaios laboratoriais, como nas reações de oxirredução para detecção da glicosúria. Objetivo: Este trabalho tem como objetivo avaliar a interferência do ácido ascórbico na detecção de glicosúria pelo método de química seca por meio do uso de tiras reagentes. Materiais e métodos: Amostras de urina foram avaliadas no analisador da marca Clinitek Atlas (Siemens Healthcare Diagnostics Inc., EUA). Foram selecionadas quatro amostras de urina com diferentes concentrações de glicose: $100 \mathrm{mg} / \mathrm{dl}, 250 \mathrm{mg} / \mathrm{dl}, 500 \mathrm{mg} / \mathrm{dl} \mathrm{e} 1.000 \mathrm{mg} / \mathrm{dl}$. Para cada concentração de glicose foram criadas cinco alíquotas, adicionando-se uma solução de ácido ascórbico $200 \mathrm{mg} / \mathrm{dl}$, suficiente para obter uma concentração final de ácido ascórbico de $20 \mathrm{mg} / \mathrm{dl}$ no primeiro tubo, de $50 \mathrm{mg} / \mathrm{dl}$ no segundo tubo, de $270 \mathrm{mg} / \mathrm{dl}$ no terceiro tubo, de $1.000 \mathrm{mg} / \mathrm{dl}$ no quarto tubo e de $2.000 \mathrm{mg} / \mathrm{dl}$ no quinto tubo. Após essa adição, as amostras foram novamente avaliadas no analisador Clinitek Atlas. Resultados: Nas amostras com concentração de 20 mg/dl de ácido, não se evidenciou interferência. Nas concentrações iguais e acima de $50 \mathrm{mg} / \mathrm{dl}$, a interferência do ácido ascórbico se fez presente, sendo que o fato foi caracterizado pelos resultados falso negativos para detecção da glicose urinária. Conclusão: Os resultados demonstraram a interferência do ácido ascórbico no método da química seca (tiras reagentes), subestimando o nível de glicose urinária.

\section{abstract}

Introduction: Ascorbic acid (vitamin C) is commonly used as a vitamin supplement. It is a water soluble vitamin, which is excreted through urine and may interfere in laboratory tests as well as redox reactions for urinary glucose detection. Objective: This study aims to assess ascorbic acid interference in glycosuria detection by dry chemistry method (reagent strips). Materials and methods: Urine samples were evaluated by using Clinitek Atlas chemistry analyzer (Siemens Healthcare Diagnostics Inc., USA). Four urine samples with different glucose concentrations were selected: $100 \mathrm{mg} / \mathrm{dl}, 250 \mathrm{mg} / \mathrm{dl}, 500 \mathrm{mg} / \mathrm{dl}$ and $\geq 1,000 \mathrm{mg} / \mathrm{dl}$. 5 aliquots were created for each glucose concentration and a solution of ascorbic acid $200 \mathrm{mg} / \mathrm{dl}$ was added, sufficient to obtain a final ascorbic acid concentration of $20 \mathrm{mg} / \mathrm{dl}$ in the first tube, $50 \mathrm{mg} / \mathrm{dl}$ in the second tube, $270 \mathrm{mg} / \mathrm{dl}$ in the third tube, 1,000 mg/dl the fourth tube, and 2,000 mg/dl in the fifth tube. After the addition of ascorbic acid, the samples were reassessed by using Clinitek Atlas chemistry analyzer. Results: There was no interference at concentrations of $20 \mathrm{mg} / \mathrm{dl}$. However, there was ascorbic acid interference at concentrations higher than or equal to $50 \mathrm{mg} / \mathrm{dl}$, which was characterized by false-negative results for urinary glucose detection. Conclusion: The results corroborated the interference of ascorbic acid in dry chemistry method (reagent strips) inasmuch as it underestimates urinary glucose levels. key words

Ascorbic acid

Glycosuria

Dry chemistry

Interference

\footnotetext{
1. Médico-residente em Patologia Clínica na Divisão de Laboratório Central do Hospital das Clínicas da Faculdade de Medicina da Universidade de São Paulo (DLC/HCFMUSP).

2. Doutora em Patologia pela FMUSP; chefe da Seção Técnica de Bioquímica de Sangue da DLC/HCFMUSP.

3. Doutor em Medicina; professor assistente da disciplina de Patologia Clínica da FMUSP; diretor do Serviço de Bioquímica Clínica da DLC/HCFMUSP.
} 


\section{Introdução}

O ácido ascórbico (vitamina C) é uma vitamina solúvel em água e tem papel fundamental na síntese de colágeno, que é uma importante proteína na constituição de vários órgãos do corpo humano. Os seres humanos são incapazes de sintetizar essa vitamina, havendo, portanto, a necessidade de obtê-la por meio da dieta. Com frequência, devido às suas propriedades antioxidantes, a vitamina $\mathrm{C}$ tem sido consumida de forma abusiva por meio de suplementos vitamínicos. Efeitos deletérios desse consumo excessivo têm sido relatados na literatura ${ }^{(4,7)}$.

No laboratório clínico, a presença de vitamina C, particularmente nas amostras de urina, representa potencial fator interferente nos resultados dos exames laboratoriais ${ }^{(3)}$.

A presença de vitamina $\mathrm{C}$ na urina pode resultar em uma resposta falsamente baixa ou até mesmo negativa para a pesquisa de glicosúria que utiliza tiras reagentes ${ }^{(2)}$. O princípio das tiras reagentes para dosagem da glicosúria envolve a transformação de glicose em ácido glucônico e peróxido de hidrogênio; uma segunda enzima, a peroxidase, promove a reação do peróxido com um cromógeno para a formação de um composto oxidado de cor marrom, sendo a intensidade dessa cor diretamente proporcional à quantidade de glicose na amostra. Nesse contexto, a vitamina C (por suas conhecidas propriedades antioxidantes) pode inibir a oxidação do cromógeno e, consequentemente, resultar em leituras falso negativas.

A interferência do ácido ascórbico tem sido relatada em vários trabalhos. Barbosa e Andrade ${ }^{(1)}$ demonstraram que o consumo da vitamina interfere nas dosagens de glicemia, enquanto Martinello e Silva ${ }^{(5)}$ mostraram a interferência in vitro não só da glicose, mas também de outros analitos.

\section{Objetivo}

Este trabalho visa verificar o grau de interferência do ácido ascórbico em diferentes níveis de concentração na urina para detecção e quantificação da glicosúria.

\section{Materiais e métodos}

Foram selecionadas quatro amostras de urina cujos níveis de glicose foram previamente avaliados no analisador químico de urina Clinitek Atlas (Siemens Healthcare Diagnostics Inc., EUA) e categorizadas em concentrações de glicosúria baixa (100 mg/dl), intermediária nível I (250 $\mathrm{mg} / \mathrm{dl})$, intermediária nível II $(500 \mathrm{mg} / \mathrm{dl})$ e elevada ( $\geq$ $1.000 \mathrm{mg} / \mathrm{dl}$ ).

Para cada uma dessas amostras foram produzidas cinco alíquotas de $3 \mathrm{ml}$ em tubos devidamente identificados, conforme descrito a seguir.

- GB-1, GB-2, GB-3, GB-4 e GB-5: alíquotas da amostra com glicosúria baixa;

- GNI-1, GNI-2, GNI-3, GNI4 e GNI-5: alíquotas da amostra com glicosúria intermediária nível I;

- GNII-1, GNII-2, GNII-3, GNII4 e GNII-5: alíquotas da amostra com glicosúria intermediária nível II;

- GA-1, GA-2, GA-3, GA-4 e GA-5: alíquotas da amostra com glicosúria elevada.

- Na sequência, adicionou-se uma solução comercial de ácido ascórbico, com concentração de 200 mg/dl, em cada grupamento de tubos:

- grupo 1 (GB-1, GNI-1, GNII-1 e GA-1) - $5 \mu \mathrm{l}$ da solução de ácido ascórbico;

- grupo 2 (GB-2, GNI-2, GNII-2 e GA-2)-12,5 $\mu \mathrm{l}$ da solução de ácido ascórbico;

- grupo 3 (GB-3, GNI-3, GNII-3 e GA-3) - 67,5 $\mu \mathrm{l}$ da solução de ácido ascórbico;

- grupo 4 (GB-4, GNI-4, GNII-4 e GA-4) - $250 \mu \mathrm{l}$ da solução de ácido ascórbico;

- grupo 5 (GB-5, GNI-5, GNII-5 e GA-5) - $500 \mu \mathrm{l}$ da solução de ácido ascórbico.

Após a adição do ácido ascórbico, cada tubo foi completado com a amostra de urina correspondente até atingir o volume de $5 \mathrm{ml}$. Ao final, para cada grupamento de tubos, obtiveram-se concentrações crescentes de ácido ascórbico correspondentes a $20 \mathrm{mg} / \mathrm{dl}$ (GB-1, GNI-1, GNII-1 e GA-1), 50 mg/dl (GB-2, GNI-2, GNII-2 e GA-2), 270 mg/dl (GB-3, GNI-3, GNII-3 e GA-3), $1.000 \mathrm{mg} / \mathrm{dl}$ (GB-4, GNl-4, GNII-4 eGA-4)e2.000 mg/dl (GB-5, GNI-5, GNII-5 e GA-5).

A definição por cinco diferentes concentrações de ácido ascórbico tinha como finalidade verificar o grau de interferência em níveis abaixo e acima de $50 \mathrm{mg} / \mathrm{dl}$ de ácido ascórbico; o nível de $50 \mathrm{mg} / \mathrm{dl}$ foi escolhido por ser supostamente o valor mínimo de interferência, conforme descrito por Massey ${ }^{(6)}$.

Após essa adição, cada alíquota foi imediatamente inserida no analisador químico de urina Clinitek Atlas para avaliação do nível de glicose. 


\section{Resultados}

Os resultados das dosagens nas diferentes alíquotas, após adição de ácido ascórbico, estão descritos na Tabela a seguir.

\begin{tabular}{|c|c|}
\hline $\begin{array}{l}\text { Concentração de ácido } \\
\text { ascórbico (mg/dl) }\end{array}$ & $\begin{array}{l}\text { Glicosúria antes da adição } \\
\text { do ácido ascórbico (mg/dl) }\end{array}$ \\
\hline
\end{tabular}

$\begin{array}{cc}\text { Grupo } 1 & \\ \text { GB-1 } & 20 \\ \text { GNI-1 } & 20 \\ \text { GNII-1 } & 20 \\ \text { GA-1 } & 20\end{array}$

Grupo 2

GB-2

GNI-2

GNII-2

GA-2

Grupo 3

GB-3

GNI-3

GNII-3

GA-3

Grupo 4

GB-4

GNI-4

GNII-4

GA-4

Grupo 5

GB-5

GNI-5

GNII-5

GA-5
20

20

20

20

50

50

50

50

270

270

270

270

1.000

1.000

1.000

1.000

2.000

2.000

2.000

2.000

$\begin{aligned} & 100 \\ & 250 \\ & 500 \\ \geq & 1.000\end{aligned}$

100

250

500

$\geq 1.000$
Negativo

100

250

250

$\geq 1.000$

100

250

500

$\geq 1.000$

100

250

500

$\geq 1.000$

100

250

500

$\geq 1.000$
Negativo

Negativo

Negativo

Negativo

Negativo

Negativo

Negativo

Negativo

Negativo

Negativo

Negativo

Negativo

\section{Discussão}

As amostras às quais se acrescentou ácido ascórbico na concentração de $20 \mathrm{mg} / \mathrm{dl}$ não evidenciaram qualquer grau de interferência nos resultados da glicose. Quando a adição foi feita na concentração de $50 \mathrm{mg} / \mathrm{dl}$, os resultados observados já sugeriam algum grau de interferência na medida da glicosúria. Nessa situação, foi observado um resultado falso negativo na amostra GB-2.

A adição de ácido ascórbico na urina, em uma concentração final igual ou superior a $270 \mathrm{mg} / \mathrm{dl}$, já produziu resultados falso negativos em todos os níveis de glicosúria. Massey refere que níveis acima de $50 \mathrm{mg} / \mathrm{dl}$ de ácido ascórbico já produziriam interferência na análise da glicose, dados concordantes com nossos achados ${ }^{(6)}$.

Em relação à frequência da positividade de ácido ascórbico em amostras de urina, o estudo de Brigden et al. com 4.379 amostras evidenciou que cerca de $23 \%$ delas eram positivas para ácido ascórbico, encontrando um valor de concentração média de $37 \mathrm{mg} / \mathrm{dl}$ na casuística avaliada(3). O mesmo estudo mostrou que a ingestão oral de uma dose contendo $250 \mathrm{mg}$ de vitamina $\mathrm{C}$ gera uma concentração média urinária de $31 \mathrm{mg} / \mathrm{dl}$ de ácido ascórbico; já uma dose oral de $500 \mathrm{mg}$ eleva a concentração média para $62 \mathrm{mg} / \mathrm{dl}\left({ }^{(3)}\right.$.

Martinello e Silva ${ }^{(5)}$ sugerem que o consumo deveria ser suspenso entre 48 e 72 horas antes de os testes serem feitos. 
A suspensão prévia nesse intervalo de tempo seria suficiente para a excreção da vitamina C pela urina, evitando, assim, a eventual interferência na dosagem da glicose pelo método da química seca.

\section{Conclusão}

A presença de ácido ascórbico na urina representa um potencial interferente na avaliação da glicosúria. Nosso estudo demonstrou que níveis iguais ou superiores a $50 \mathrm{mg} / \mathrm{dl}$ de ácido ascórbico já podem produzir resultados falso negativos.

Um questionamento acerca do uso dessa substância se faz necessário para que resultados discordantes possam ser adequadamente avaliados pelo laboratório clínico.

\section{Referências}

1. BARBOSA A. C.; ANDRADE T. C. Interferência do ácido ascórbico na dosagem glicêmica. Universitas: Ciências da Saúde, Brasília, v. 6, n. 2, p. 121-30, jul./dez. 2008.

2. BERG, B. Ascorbate interference in the estimation of urinary glucose. J Clin Chem Clin Biochem, v. 24, p. 89-96, 1986.

3. BRIGDEN, M. L. et al. High incidence of significant urinary ascorbic acid concentrations in a west coast population Implications for routine urinalysis. Clin Chem, v. 38, n. 3, p. 426-31, 1992.
4. HEANEY, M. L. et al. Vitamin C antagonizes the cytotoxic effects of antineoplastic drugs. Cancer Res, v. 68 n. 19, 2008.

5. MARTINELLO, F.; SILVA, E. L. Interferência do ácido ascórbico nas determinações de parâmetros bioquímicos séricos: estudos in vivo e in vitro. J Bras Patol Med Lab, v. 39, n. 4, p. 323-34, 2003.

6. MASSEY, L. D. Seven rules for error-free urinalysis. Disponível em: <http://laboratorian.advanceweb.com/ Article/Seven-Rules-For-Error-Free-Urinalysis.aspx>. Acesso em: 8 mar. 2011.

7. MOORE, T.; WANG, Y. Hypervitaminosis A. Biochem J, v. 39, p. 222-8, 1945. 